\title{
On the Utilization of MIMO-OFDM Channel Sparsity for Accurate Positioning
}

\author{
Jani Saloranta and Giuseppe Destino \\ Centre for Wireless Communications \\ University of Oulu, \\ Oulu, Finland \\ \{jani.saloranta, giuseppe.destino\}@ee.oulu.fi
}

\begin{abstract}
Recent results have revealed that MIMO channels at high carrier frequencies exhibit sparsity structure, i.e., a few dominant propagation paths. Also channel parameters, namely angular information and propagation delay can be modelled with the physical location of the transmitter, receiver and scatters. In this paper, we leverage these features into the development of a single base-station localization algorithm, and show that the location of an unknown device can be estimated with an accuracy below a meter based on pilot signalling with a OFDM transmission. The method relies on the utilization of the "Adaptive-LASSO" optimization method, in which an $\ell_{1}$. based minimization problem is solved by adapting the sparsifying matrix (dictionary) and the sparse vector jointly. Then the location of the device is estimated from the parameters of the sparsifying matrix. Finally, the positioning method is evaluated in different channel setting utilizing a ray-tracing channel model at $28 \mathrm{GHz}$.
\end{abstract}

\section{INTRODUCTION}

For the last decade, location-awareness has been the paradigm pursued in many researches on sensor networks, Internet-of-Things (IoT), and more generally, positioning applications. [1] With 5G, the fifth-generation of mobile system [2], [3], there is a surge of interests towards location-awareness for mobile communications. In contrast to the past, new technologies such as millimeter-wave (mmWave) and three dimensional (3D) multiple-input and multiple-output (MIMO) quest for precise and real-time user-equipment (UE) location information for the development of highly directional [4] and dynamic beamforming [5], [6].

However, if positioning is based on dedicated technologies such as Global Positioning System (GPS), Wi-Fi, ultra-wide band (UWB) and Bluetooth, then the cost (data traffic, latency and energy consumption) of interfacing the mobile system with external technologies can be prohibitive. For this reason, it is desirable to directly develop a mobile-network based positioning although some technology challenges has to be addressed to achieve high accuracy and lightweight integration.

In this paper, we propose a novel positioning approach based on MIMO-orthogonal frequency-division multiplexing (OFDM) transmission at the mmWave frequency band. Also, we consider a single-base station solution in order to reduce the energy consumption due to a multi-point cooperation [7].

The key of the proposed technique is to leverage the sparsity of a mmWave channel [8], i.e., that signal propagates only through a few paths, into the estimation process.
More specifically, we rely on a multidimensional sparse representation of the channel obtained with the adaptive-least absolute shrinkage and selection operator (A-LASSO) [9], [10] algorithm and develop a channel-based location mapping and a line-of-sight (LOS)-based positioning via a Gaussian mixture model (GMM) classification technique. Simulation results have shown that the proposed approach can provide sub-meter accuracy with relatively small number of antennas and signal bandwidth, though only in LOS channel conditions.

The rest of the paper is organized as follows. In Sections II, we present the system model. In Section III, we explain the position estimation problem within a single base-station scenario and detail the technique to solve the problem. In Section IV, results of the proposed positioning method are shown in LOS and non-line-of-sight (NLOS) scenario via raytracing -based simulation for an office environment. Finally, in Section V, concluding remarks are given.

\section{System Model}

We consider a 3D positioning problem of a UE using one small-cell base-station (BS). We assume an up-link transmission where the UE sends an OFDM pilot-signal with bandwidth $B_{p}<B$, where $B$ is the maximum transmission bandwidth, e.g., $200 \mathrm{MHz}$. The OFDM signal is designed with $N_{\text {FFT }}$ subcarriers. The number of transmit and receive antennas are $N=1$ and $M$, respectively. The BS location is known $a$ priori and given by $\mathbf{b} \in \mathbb{R}^{3}$, whereas the UE position, denoted by $\mathbf{x} \in \mathbb{R}^{3}$, is yet to be determined.

In order to obtain a 3D location estimate, the $\mathrm{BS}$ is equipped with two-dimensional antenna array, e.g., a uniform rectangular array (URA) deployed in the $y z$-plane, with a sufficiently large number of elements. Also, to minimize the cost and the energy consumption of the BS transceiver, the hybrid analog-digital architecture depicted in Figure 1 [8] is utilized. We consider $M_{B B}$ independent digital paths, each one comprising an analog-to-digital converter, amplifiers, a phaseshifter based analog beamformer and $m$ antenna elements. The digital beamformer is modelled with a matrix $\mathbf{W} \in \mathbb{C}^{M \times M_{t}}$ where $M_{t}$ is the number of combining vectors.

In the frequency domain, the received signal is given by

$$
\mathbf{Y}=\sqrt{\rho} \mathbf{W}^{\mathrm{H}} \mathbf{A}^{\mathrm{H}}(\mathbf{H X}+\mathbf{N}),
$$




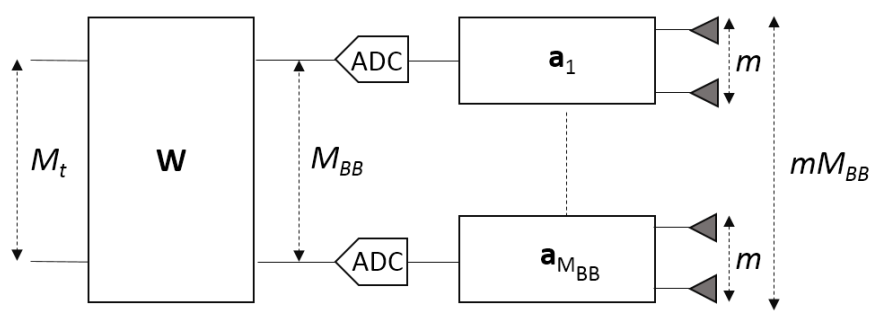

Figure 1. Analog-digital architecture of the receiver

where $(\cdot)^{\mathrm{H}}$ is the transpose conjugate, $\rho$ is the average received power, A $\in \mathbb{C}^{M \times M_{B B}}$ is the "total" analog beamformer matrix that is block-diagonal with the $i$-th block given by the vector $\mathbf{a}_{i} \in \mathbb{C}^{m}$ and $\left|a_{j i}\right|=1, \mathbf{H} \triangleq\left[\mathbf{h}_{1}, \cdots, \mathbf{h}_{N}\right]$ is the MIMO channel matrix, $N<N_{\mathrm{FFT}}$ is the number of pilot-subcarriers, $\mathbf{X} \in \mathbb{C}^{N \times N}$ is a diagonal matrix where the diagonal elements are the pilot symbols, and $\mathbf{N} \in \mathbb{C}^{M_{t} \times N}$ is the noise matrix with $n_{i j} \sim \mathcal{C N}(0,1)$ as a complex-Gaussian random variable.

At the $n$-th subcarrier, the channel vector is modelled as a frequency-selective wideband clustered multiple-input and single-output (MISO) channel, in which $N_{t}$ is the number of clusters and $N_{q}$ is the number of propagation paths per cluster [11]. Mathematically, the MISO channel $\mathbf{h}_{n}$ is given by

$$
\mathbf{h}_{n}=\sqrt{\frac{M}{N_{t} N_{q} N_{\mathrm{FFT}}}} \sum_{t=1}^{N_{t}} \sum_{q=1}^{N_{q}} \alpha_{q, t} \mathbf{a}_{R}\left(\phi_{q, t}, \theta_{q, t}\right) e^{-j 2 \pi \frac{n \tau_{q, t} B}{N_{\mathrm{FFT}}}},
$$

where the index $(\cdot)_{q, t}$ refers to $q$-th path of the $t$-th cluster, $\phi_{q, t}, \theta_{q, t}$ are the azimuth and elevation of the angle of arrival, $\tau_{q, t}$ is path-delay, $\alpha_{q, t} \sim \mathcal{C N}\left(0, \sigma_{q, t}^{2}\right)$ is a complex Gaussian random variable modelling the complex gain of a path with energy $\sigma_{q, t}^{2}$ and $\mathbf{a}_{R}(x, y)$ is the receiving steering vector

$$
\mathbf{a}_{R}(x, y)=e^{-j \frac{2 \pi}{\lambda} \mathbf{k}^{\mathrm{T}}(x, y) \mathbf{P}},
$$

where $(\cdot)^{\mathrm{T}}$ indicates transpose, $\mathbf{P} \in \mathbb{R}^{3 \times M}$ is the matrix containing the (3D) location of antenna elements ${ }^{1}$ and $\mathbf{k}(x, y) \triangleq$ $[\cos (x) \cos (y), \sin (x) \cos (y), \sin (y)]^{\mathrm{T}}$.

\section{Single BS Positioning}

Using a single reference point, namely the location of a base-station, $x$ can be computed as a function of the LOS path-delay, azimuth and elevation angle of arrival as

$$
\mathbf{x}=\mathbf{b}+r[\cos (\bar{\phi}) \cos (\bar{\theta}), \sin (\bar{\phi}) \cos (\bar{\theta}), \sin (\bar{\theta})]^{\mathrm{T}},
$$

with $\bar{\phi}=\phi_{\bar{q}, \bar{t}}, \bar{\theta}=\theta_{\bar{q}, \bar{t}}$ and, finally, $r=c \tau_{\bar{q}, \bar{t}}$, where $c$ equals to the speed-of-light and $(\cdot)_{\bar{q}, \bar{t}}$ refers to the LOS path index.

More generally, we can formulate a position estimation problem based on the received signal $\mathbf{Y}$ as

$$
(\hat{\mathbf{x}}, \hat{\boldsymbol{\Delta}}, \hat{\boldsymbol{\beta}})=\min _{\substack{\boldsymbol{\beta} \in \mathbb{C}^{L}, \mathbf{x} \in \mathbb{R}^{3} \\ \boldsymbol{\Delta} \in \mathbb{R}^{3 \times L}}}\left\|\mathbf{Y}-\mathbf{W}^{\mathrm{H}} \mathbf{A}^{\mathrm{H}} \mathcal{H}(\mathbf{x}, \boldsymbol{\Delta}) \mathbf{X}\right\|_{2}^{2}
$$

\footnotetext{
${ }^{1}$ Coordinates are given with respect to the location of one element set as
}

where

$$
\begin{gathered}
\mathcal{H}(\mathbf{x}, \boldsymbol{\Delta})=\left[\sum_{l=1}^{L} \beta_{l} \tilde{\mathbf{a}}_{R}\left(\mathbf{x}, \boldsymbol{\delta}_{l} \mid 1\right), \cdots, \sum_{l=1}^{L} \beta_{l} \tilde{\mathbf{a}}_{R}\left(\mathbf{x}, \boldsymbol{\delta}_{l} \mid N\right)\right], \\
\tilde{\mathbf{a}}_{R}\left(\mathbf{x}, \boldsymbol{\delta}_{q, t} \mid n\right)=e^{-j \frac{2 \pi}{\lambda} \frac{\left(\mathbf{x}-\mathbf{b}+\delta_{q, t}\right)^{\mathrm{T}}}{\left.\| \mathbf{x}-\mathbf{b}+\boldsymbol{\delta}_{q, t}\right) \|_{2}} \mathbf{P}} e^{-j 2 \pi \frac{n B\left\|\mathbf{x}-\mathbf{b}+\boldsymbol{\delta}_{q, t}\right\|_{2}}{c N_{\mathrm{FFT}}}},
\end{gathered}
$$

and $\Delta \in \mathbb{R}^{3 \times L}$ is a matrix in which the $l$-th column is given by a location-shift $\boldsymbol{\delta}_{l}$ and $L$ is the number of components in which each channel vector $\mathbf{h}_{n}$ is decomposed.

This minimization problem is very difficult to solve since the terms in the summation (6) are given by the product of the variable $\beta_{l}$ and the non-convex function $\tilde{\mathbf{a}}_{R}\left(\mathbf{x}, \boldsymbol{\delta}_{l} \mid n\right)$ and, additionally, the solution may depend on the selection of $L$. In order to circumvent these challenges, we propose an indirect approach, in which we exploit the channel parametrization given in [9], [10] to generate a set of location images $\left\{\tilde{\mathbf{x}}_{l}\right\}$, with $\tilde{\mathbf{x}}_{l} \triangleq \mathbf{x}+\boldsymbol{\delta}_{l}$, and estimate $\mathbf{x}$ based on the cluster of images associated to the LOS link.

\section{A. Mapping from MIMO-OFDM Channel to Location Images}

Let us consider the vectorized form of the MIMO-OFDM channel $\mathbf{H}$, i.e., $\mathbf{h} \triangleq \operatorname{vec}(\mathbf{H})$ and express $\mathbf{h}$ with the linear model $\mathbf{h}=\mathbf{\Psi} \mathbf{z}$, where $\boldsymbol{\Psi} \in \mathbb{C}^{N_{\mathrm{FFT}} M \times L}$ is referred to as "dictionary" and $\mathbf{z} \in \mathbb{C}^{L}$ is the representation of $\mathbf{h}$ in $\Psi$ [9], [10]. A column vector of the dictionary, hereafter referred to as atom, corresponds to the vectorization of a multidimensional spatial-temporal discrete Fourier frequency. More specifically, we compute $\boldsymbol{\Psi}=\mathcal{D}(\boldsymbol{\Xi})$, where $\boldsymbol{\Xi} \in \mathbb{R}_{+}^{U \times L}$ is the "dictionary variable" and $\mathcal{D}(\boldsymbol{\Xi})$ is the "dictionary function" defined as

$$
\mathcal{D}(\boldsymbol{\Xi}) \triangleq\left[\bigotimes_{i=1}^{U} \mathbf{v}\left(\xi_{i 1}, K_{i}\right), \cdots, \bigotimes_{i=1}^{U} \mathbf{v}\left(\xi_{i L}, K_{i}\right)\right],
$$

where $\xi_{i j}$ is the $i j$-th element of $\Xi, \bigotimes$ indicates the "total" Kronecker product of $U$ vectors and $\mathbf{v}(x, y) \in \mathbb{C}^{y}$ is the discrete complex-frequency

$$
\mathbf{v}(x, K)=\left[1, \cdots, e^{-j 2 \pi x(K-1)}\right]^{\mathrm{T}},
$$

with $x \in[0,1)$ and $K \in \mathbb{N}$.

Inside the dictionary function (8), the parameter $U$ refers to the number of dimensions used to represent the spatialtemporal discrete frequencies. For instance, if the MIMOOFDM channel can be represented with a bi-dimensional spatial frequency and a mono-dimensional Fourier frequency, $U$ equals to three. The value of $K_{i}$, instead, depends on the specific problem setting. For instance, with $N_{\text {FFT }}$ subcarriers, the $K_{i}$ parametrizing the mono-dimensional Fourier frequency equals to $N_{\mathrm{FFT}}$. Whereas, if we consider a URA, the values of the $K_{i}$ modelling the spatial-frequency are given by number of rows and columns of the antenna structure.

As shown in [9], [10], the $l$-th column vector of $\boldsymbol{\Xi}$, denoted by $\xi_{l}$, relates to the angle of $\operatorname{arrival}^{2}$ and time delay of a channel path. Therefore, each $\xi_{l}$ can be mapped to a location

${ }^{2}$ If multiple antenna system is used for transmission, the vector boldsymbol $\xi_{l}$ will include also elements related to the angle of departure. 
$\tilde{\mathbf{x}}_{l}$. For instance, following the aforementioned example of URA, the map function is given by

$$
\tilde{\mathbf{x}}_{l}=\mathbf{b}+\left[\begin{array}{c}
\frac{c \sqrt{1-u_{2 l}^{2}}}{\Delta_{f}} \cos \left(\operatorname{asin}\left(u_{1 l}\right) \sec \left(\sin \left(u_{2 l}\right)\right)\right) u_{3 l} \\
-\frac{c \sqrt{1-u_{2 l}^{2}}}{\Delta_{f}} \sin \left(\operatorname{asin}\left(u_{1 l}\right) \sec \left(\sin \left(u_{2 l}\right)\right)\right) u_{3 l} \\
-\frac{c \sqrt{1-u_{2 l}^{2}}}{\Delta_{f}} u_{2 l} u_{3 l}
\end{array}\right],
$$

where $\Delta_{f}=B / N_{\mathrm{FFT}}$ and

$$
u_{i l}= \begin{cases}2 \xi_{i l}, & 0 \leq \xi_{i l}<0.5 \\ 2 \xi_{i l}-2, & 0.5 \leq \xi_{i l}<1\end{cases}
$$

In practice, the dictionary variable $\boldsymbol{\Xi}$ is not known, but it can be estimated from the received signal $\mathbf{Y}$ with the A-LASSO technique [9], [10] that solves the optimization problem

$$
\begin{gathered}
(\hat{\mathbf{z}}, \hat{\boldsymbol{\Xi}})=\min _{\substack{\mathbf{z} \in \mathbb{C}^{L} \\
\boldsymbol{\Xi} \in \mathbb{R}^{U} \times}} \lambda\|\mathbf{z}\|_{1}+\frac{1}{2}\left\|\mathbf{y}-\left(\mathbf{X} \mathbf{S}_{f}^{\mathrm{T}} \otimes \mathbf{W}^{\mathrm{H}} \mathbf{A}^{\mathrm{H}}\right) \mathbf{\Psi} \mathbf{z}\right\|_{2}^{2} \\
\text { s.t. } \quad \boldsymbol{\Psi}=\mathcal{D}(\boldsymbol{\Xi}), \quad \underline{\xi_{i}} \leq \xi_{i j}<\bar{\xi}_{i}, \forall i j
\end{gathered}
$$

where $\|\cdot\|_{q}$ is the $q$-norm, $\otimes$ denotes the Kronecker product, $\mathbf{y}=\operatorname{vec}(\mathbf{Y}), \nu$ is a parameter that controls the sparsity $\left(\approx 1 / \sqrt{N_{\mathrm{FFT}}}\right), \xi_{i}$ and $\bar{\xi}_{i}$ are the minimum and the maximum values of $\xi_{i j}$ that can be simply set $\xi_{i}=0$ and $\bar{\xi}_{i}=1$, and $\mathbf{S}_{f} \in \mathbb{R}^{N_{\mathrm{FFT}} \times N}$ is the "frequency selection" matrix ${ }^{3}$ used to select the the pilot frequency from the $N_{\mathrm{FFT}}$ subcarriers.

\section{B. LOS Classification and Positioning}

Once the variables from the dictionary have been mapped to a set of UE images $\tilde{\mathbf{x}}_{l}$, the next operation is to classify them into LOS and NLOS points. In this regard, the key is the theoretical underpinning of the dictionary optimization performed in the A-LASSO algorithm.

In fact, the dictionary atoms are computed based on a resampling-randomization strategy that follows the heuristic that the higher the value of $\left|z_{i}\right|$, i.e. the $i$-th element of $\mathbf{z}$, the higher is the "importance" of the $i$-th atom. Subsequently, the A-LASSO yields a dictionary with a support concentrated around the most dominant spatial-temporal frequency representing the channel. In other words, the algorithm provides a denser set of $\tilde{\xi}_{l}$ at those values corresponding to the strongest channel path, typically the LOS path when the signal is transmitted at high carrier frequency. [4], [12]

Therefore, the classification method consists of clustering the set of $\tilde{\xi}_{l}$, or equivalently the locations $\tilde{\mathbf{x}}_{l}$ 's, and consider the LOS cloud as the cluster with the highest node density. To do so, we propose a classification based on a GMM that solves the optimization problem $[13]^{4}$

$$
\left\{\hat{\boldsymbol{\mu}}_{i}, \hat{\boldsymbol{\Sigma}}_{i}, \hat{\gamma}_{i}\right\}=\max _{\left\{\hat{\boldsymbol{\mu}_{i}}, \hat{\boldsymbol{\Sigma}}_{i}, \hat{\gamma}_{i}\right\}} \prod_{l=1}^{L} \sum_{i=1}^{K_{g}} \gamma_{i} p\left(\tilde{\mathbf{x}}_{l} \mid \boldsymbol{\mu}_{\boldsymbol{i}}, \boldsymbol{\Sigma}_{\boldsymbol{i}}\right),
$$

\footnotetext{
${ }^{3}$ The $n$-th column of $\mathbf{S}_{f}$ has only one non-zero element, i.e., 1 , at the row-index corresponding to the $n$-th subcarrier index.

${ }^{4}$ We utilize a standard Expectation-Maximization (EM) method to solve the maximization problem.
}

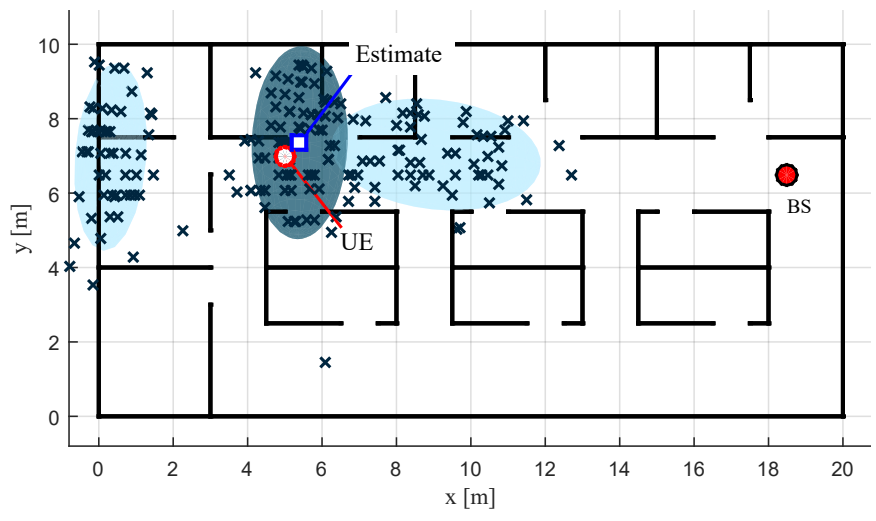

Figure 2. The indoor scenario used in simulations with the illustration of Gaussian mixture components.

where

$$
p\left(\tilde{\mathbf{x}}_{l} \mid \boldsymbol{\mu}_{\boldsymbol{i}}, \boldsymbol{\Sigma}_{\boldsymbol{i}}\right)=\frac{1}{\sqrt{(2 \pi)^{3}\left|\boldsymbol{\Sigma}_{i}\right|}} e^{-\frac{1}{2}\left(\tilde{\mathbf{x}}_{l}-\boldsymbol{\mu}_{i}\right)^{\mathrm{T}} \boldsymbol{\Sigma}_{i}^{-1}\left(\tilde{\mathbf{x}}_{l}-\boldsymbol{\mu}_{i}\right)},
$$

and $K_{g}$ is the number of Gaussian components, which can be set to small (e.g., 2 or 3) based on priori information of the environment as well as the carrier frequency (the higher the frequency, the lower is $K_{g}$ ).

Notice that we prefer to use the set $\left\{\tilde{\mathbf{x}}_{l}\right\}$ rather than $\left\{\boldsymbol{\xi}_{l}\right\}$ as the dimensions of the data points is fixed to three (two if we perform two-dimensional positioning). Finally, to perform positioning, we select the Gaussian component with the highest cluster weight $\gamma_{i}$ and obtain the estimate of the UE location related to that component as

$$
\hat{\mathbf{x}}=\hat{\boldsymbol{\mu}}_{s}, \quad \text { with } s=\arg \max \gamma_{i} \text {. }
$$

For the sake of illustration, Figure 2 shows an outcome of the estimation process. The cross refer to the locations of the image points $\tilde{\mathbf{x}}_{l}$ 's obtained from the mapping of the dictionary variables $\xi$ 's. The ellipses are derived from the estimated covariances utilizing the GMM clustering, the real location of the UE is depicted with the red circle and the blue square is the estimated location.

\section{Simulation Results}

We consider an indoor scenario, namely, an office environment, and investigate the performance of the positioning algorithm in LOS and NLOS channel conditions. Figure 3(a) and Figure 3(b)) show the location of the UE (transmitter) and BS (receiver) in the two aforementioned settings.

The MIMO-OFDM link is operating at $28 \mathrm{GHz}$ carrier frequency and the total channel bandwidth is $B=200 \mathrm{MHz}$. The OFDM symbol is designed with $N_{\text {FFT }}=2048$ subcarriers, of which only $N<N_{\mathrm{FFT}}$ are used for pilot transmission. The ray-trace simulator models the propagation paths using 4 interactions of specular reflection and diffraction.

We consider a URA at the receiver with $8 \times n, n=$ $\{1, \ldots, 8\}$ elements deployed in the $y z$-plane and equispaced 


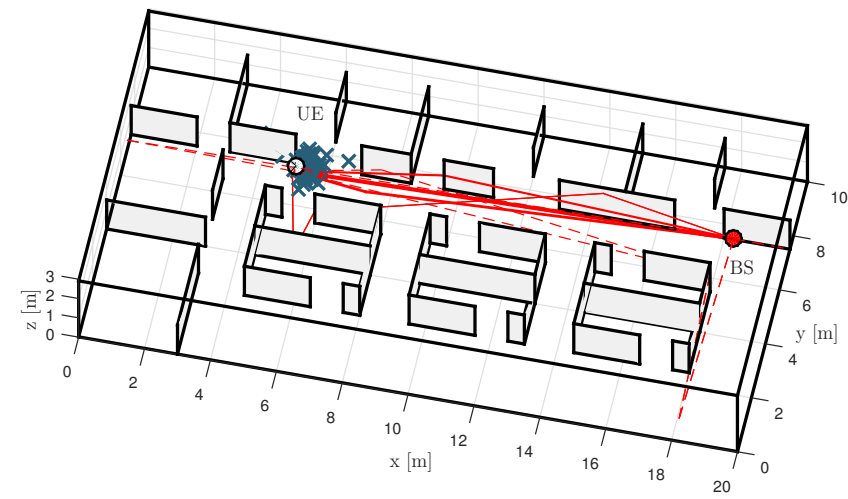

(a) Office environment - LOS scenario.

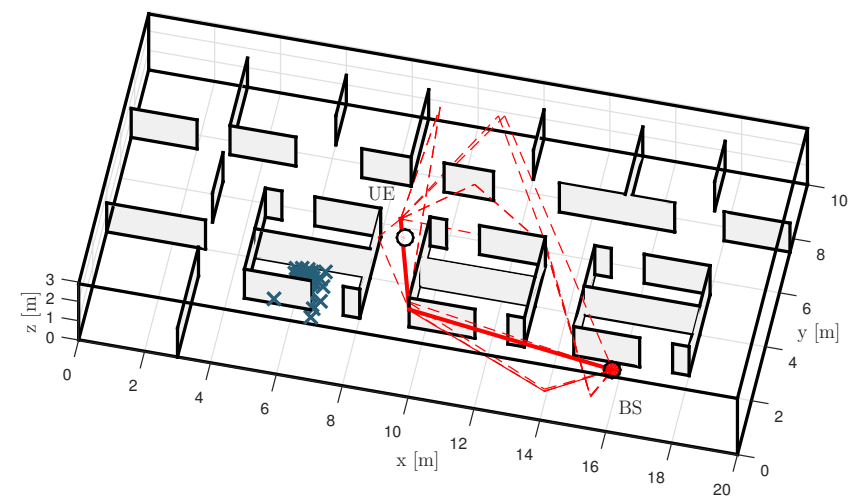

(b) Office environment - NLOS scenario.

Figure 3. Example of the simulation scenario with receiver (RX), transmitter (TX) and ray-trace paths (red lines) shown (a) in LOS scenario and (b) in NLOS scenario. The receiver and the transmitter heights are $1.8 \mathrm{~m}$ and $0.4 \mathrm{~m}$, respectively. The plots also illustrate the position estimates (the " $\mathrm{x}$ " marker).

by $\lambda / 2$. Furthermore, we assume that the UE can transmit only within a spherical-sector defined by the azimuth and elevation intervals $[-\pi / 2, \pi / 2]$ (model with a back-reflector), whereas the $\mathrm{BS}$ can receive within the azimuth and elevation sectors $[-\pi / 4, \pi / 4]$. Using this realistic sectorization we obtain a channel with a few number of paths as, for instance, illustrated in Figure 3, where solid and dashed lines refer to the effective and removed channel rays, respectively. Subsequently, the channel is very sparse.

We evaluate the root-mean-square error (RMSE) on the estimation of the transmitter location as a function of the signal-to-noise ratio (SNR), the URA structure and the pilot bandwidth $B_{p}$. Also, we investigate the impact of the hybrid analog-digital architecture on the positioning performance by testing two different beamforming modes, namely, the fullcycle and half-cycle beam switching [10].

Figure 4 shows the position error as a function the SNR with different antenna structure and beamforming strategies. Generally, it is shown that the localization error improves with the SNR in all simulation settings, but the $8 \times 2$ with half-cycle. More specifically, it is noticed that a sub-meter accuracy can be achieved at the high-SNR with a $8 \times 8$ and $8 \times 4$ URAs.

Also, as expected, the proposed algorithm can not meet the sub-meter requirements in NLOS (with LOS blockage)

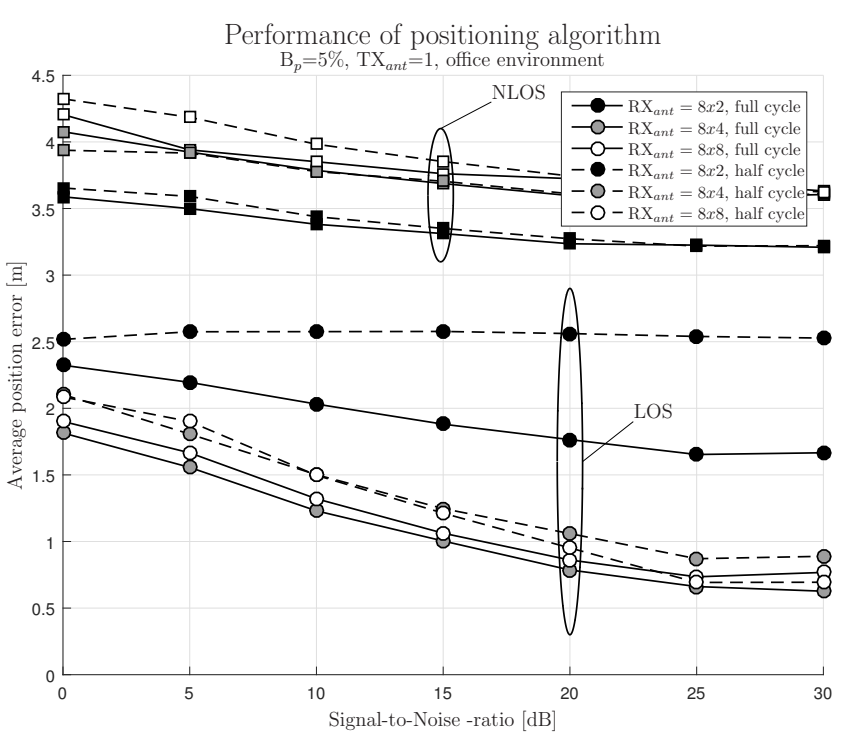

Figure 4. Comparison of the channel estimation algorithms as function of SNR.

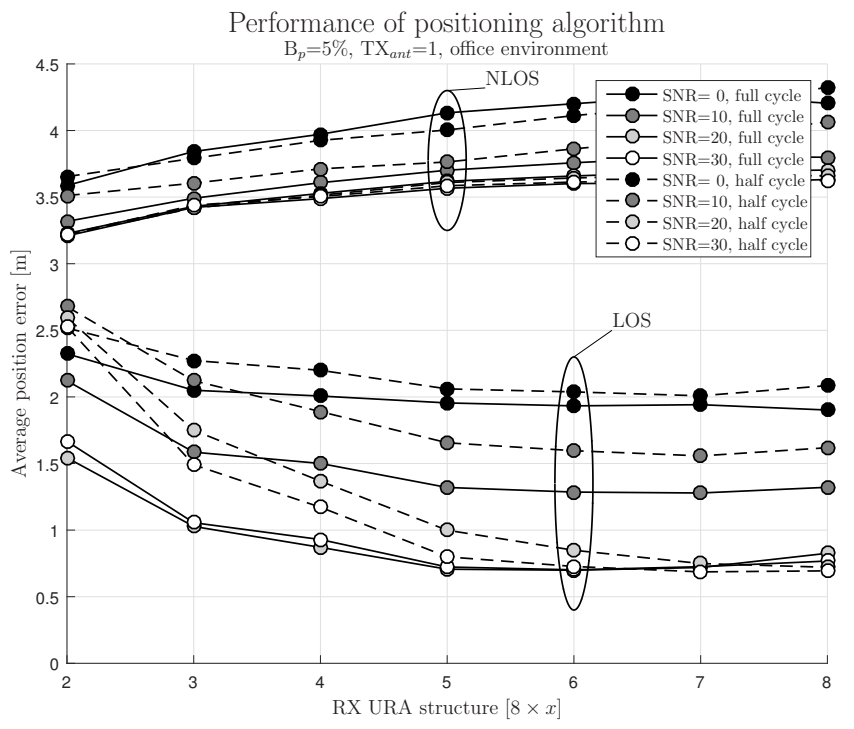

Figure 5. Comparison of the channel estimation algorithms as function of receiver antennas (RX URA structure).

channel conditions as the current estimation model does not account for path reflections. In this regard, the authors are currently investigating a solution to this problem.

Figure 5 shows the performance as a function of number of antenna elements in URA structure. The target sub-meter accuracy is already met with $8 \times 4$ elements with full and reduced cycle settings after $20 \mathrm{~dB}$ SNR. Also, it can be noticed that there is not a significant gain by increasing the number of elements in the $z$-direction. Most probably, this is a specific result related to choice of the UE and BS locations.

Finally, Figure 6 shows the location error as a function of the SNR and signal bandwidth. In this study, the antenna structure is fixed with $8 \times 8$ elements. The most important outcome is that a position error less than one meter can be achieved 


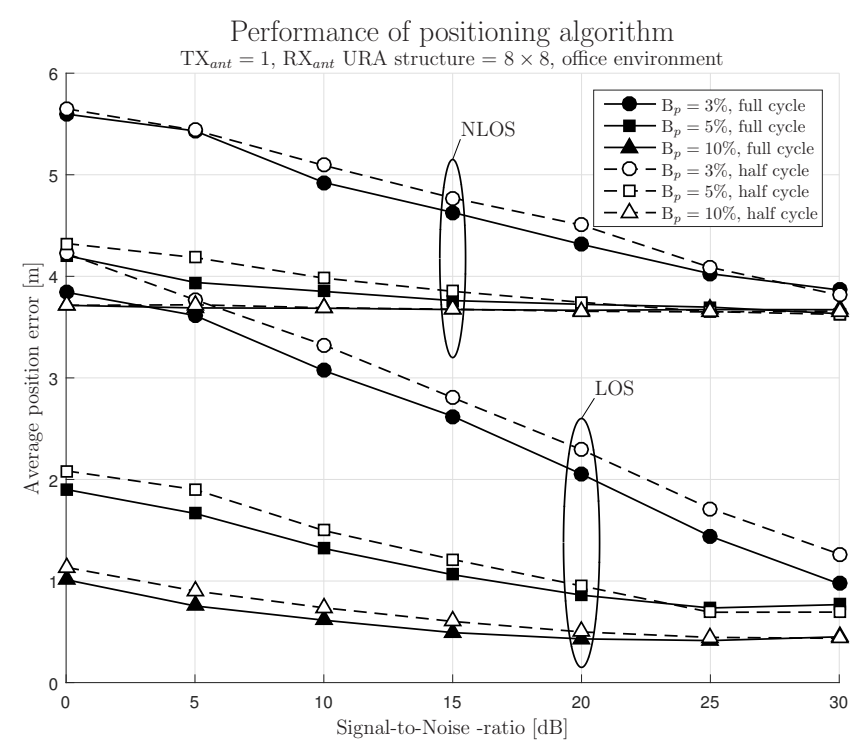

Figure 6. Comparison of the channel estimation algorithms as function of SNR.

at the low-SNR régime, for instance with $B_{p}=20 \mathrm{MHz}$ $(10 \% B)$. The reason is two-fold. First, the dictionary optimization performed in the A-LASSO algorithm operates as a de-noising technique. Thus, the set of location images is already cleared from many outliers due to the noise. Second, as the channel is sparse and, essentially characterized by a strong LOS component, the LOS cluster can be easily identified at the low-SNR, too.

\section{CONCLUSIONS}

In this paper, we proposed a novel positioning method with a single base-station using mmWave transmissions and large antenna arrays. The key is the multidimensional sparse representation of the channel obtained via the A-LASSO algorithm, which enables a direct mapping of channel parameters to expected target locations. Simulation results have shown that the proposed approach can provide sub-meter accuracy with relatively small number of antennas and signal bandwidth, though only in LOS channel conditions.

Future work shall investigate a generalization of the proposed method to account for NLOS paths as well as to provide confidence bounds to the estimated location by using the theoretical underpinning of the Circular-based Interval SMACOF (CIS) algorithm [14].

\section{ACKNOWLEDGMEnT}

The research was funded in part by Nokia and Huawei.

\section{REFERENCES}

[1] Y. Gu, A. Lo, and I. Niemegeers, "A survey of indoor positioning systems for wireless personal networks," IEEE Commun. Surveys Tuts., vol. 11, no. 1, pp. 13-32, First Quarter 2009.

[2] J. G. Andrews, S. Buzzi, W. Choi, S. V. Hanly, A. Lozano, A. C. K. Soong, and J. C. Zhang, "What will 5g be?" IEEE J. Sel. Areas Commun., vol. 32, no. 6, pp. 1065-1082, Jun. 2014.

[3] S. Zhang, X. Xu, Y. Wu, and L. Lu, "5g: Towards energy-efficient, lowlatency and high-reliable communications networks," in Proc. IEEE Int. Conf. Commun. Systems, Nov. 2014, pp. 197-201.

[4] S. Rangan, T. S. Rappaport, and E. Erkip, "Millimeter-wave cellular wireless networks: Potentials and challenges," Proc. IEEE, vol. 102, no. 3, pp. 366-385, Mar. 2014

[5] R. D. Taranto, S. Muppirisetty, R. Raulefs, D. Slock, T. Svensson, and H. Wymeersch, "Location-aware communications for 5g networks: How location information can improve scalability, latency, and robustness of 5g," IEEE Signal Process. Mag., vol. 31, no. 6, pp. 102-112, Nov. 2014.

[6] L. Muppirisetty, H. Wymeersch, J. Karout, and G. Fodor, "Locationaided pilot contamination elimination for massive mimo systems," in Proc. IEEE Global Telecommun. Conf. Workshops, Dec. 2015.

[7] S. Jeong, O. Simeone, A. Haimovich, and J. Kang, "Beamforming design for joint localization and data transmission in distributed antenna system," IEEE Transactions on Vehicular Technology, vol. 64, no. 1, pp. 62-76, Jan 2015.

[8] S. Han, C.-L. I, Z. Xu, and C. Rowell, "Large-scale antenna systems with hybrid analog and digital beamforming for millimeter wave 5g," IEEE Commun. Mag., vol. 53, no. 1, pp. 186-194, Jan. 2015.

[9] G. Destino, M. Juntti, and S. Nagaraj, "Leveraging sparsity into massive mimo channel estimation with the adaptive-lasso," in Proc. IEEE Global Signal Process. Conf., Dec. 2015.

[10] G. Destino, J. Saloranta, M. Juntti, and S. Nagaraj, "Robust 3D MIMOOFDM channel estimation with hybrid Analog-Digital architecture (submitted)," in Proc. European Sign. Process. Conf., Budapest, Hungary, Aug. 2016.

[11] O. El Ayach, S. Rajagopal, S. Abu-Surra, Z. Pi, and R. W. Heath, "Spatially sparse precoding in millimeter wave MIMO systems," IEEE Trans. Wireless Commun., vol. 13, no. 3, pp. 1499-1513, Mar. 2014.

[12] Y. Niu, Y. Li, D. Jin, L. Su, and A. V. Vasilakos, "A survey of millimeter wave communications (mmwave) for $5 \mathrm{~g}$ : opportunities and challenges," Wireless Networks, vol. 21, no. 8, pp. 2657-2676, 2015.

[13] C. Bishop, Pattern Recognition and Machine Learning, ser. Information science and statistics. Springer, 2013.

[14] J. Saloranta, D. Macagnano, and G. Abreu, "Algebraic confidence in positioning problems," in Conference Record of the Forty Seventh Asilomar Conference on Signals, Systems and Computers (ASILOMAR), 2013, Nov. 2013 